\title{
The most oxygen-poor planetary nebula: AGB nucleosynthesis at low metallicities
}

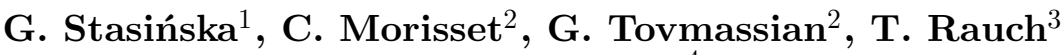 \\ and T. Decressin ${ }^{4}$
}

${ }^{1}$ LUTH, Observatoire de Paris, CNRS, Université Paris Diderot; Meudon, France

${ }^{2}$ Instituto de Astronomia, Universidad Nacional Autonoma de Mexico, Mexico, Mexico ${ }^{3}$ Institute for Astronomy and Astrophysics, Eberhard Karls University, Tuebingen, Germany

${ }^{4}$ Argelander Institute for Astronomy (AIfA), Auf dem Hügel 71, D-53121 Bonn, Germany

\begin{abstract}
TS 01 is an exceptional planetary nebula (PN) in the Galactic halo: it is the most oxygen-poor and has a double-degenerate core with mass close to $1.4 \mathrm{M}_{\odot}$, possibly a Supernova Ia progenitor. With data from the far UV to the IR we can pin down the abundances of half a dozen of elements. The oxygen abundance is by $1.9( \pm 0.3)$ dex lower than in the Sun. Standard AGB models with appropriate mass and metallicity cannot explain the observed chemical composition. We find that additional mixing, induced by stellar rotation and/or by the presence of the close companion can explain most of the features of the abundance pattern in TS 01.
\end{abstract}

Keywords. ISM: planetary nebulae: individual - ISM: abundances - Stars: AGB and postAGB - Stars: binaries

\section{Introduction}

PN G 135.9+55.9/SBS 1150+599A, now referred to as TS 01, has an oxygen abundance much lower than any other known PN. Its stellar core is a double degenerate close binary system with period $3.9 \mathrm{~h}$ and total mass close to the Chandrasekhar limit. The story of its discovery is presented in Tovmassian et al. (2001, 2004). Here we summarize our present knowledge on its chemical composition and its interpretation in the light of current AGB theory. A full presentation of this and other aspects of our work regarding the past and future of this object can be found in Stasińska et al. (2009) and Tovmassian et al. (2009).

Standard methods for abundance determinations in PNe cannot be used for TS 01, since the electron temperature cannot be obtained from the observations. Instead, photoionization models have to be constructed to make use of the largest possible set of observational constraints. Table 1 presents the status of the abundances derived for TS 01 since 2001. These abundances were based on an increasing set of observations, improving both in quality and wavelength coverage. The study of Tovmassian et al. (2001) relied on optical spectra where the only line from a heavy element was [O III] $\lambda 5007$. Richer et al. (2002) and Jacoby et al. (2002) independently acquired deeper spectra extending more to the ultraviolet and showing the $[\mathrm{NeV}] \lambda 3426$ line. As a matter of fact, both sets of line intensities were later shown to be incorrect. Péquignot \& Tsamis (2005) made the best use of the merged optical data sets, but did not include in their analysis the ultraviolet FUSE and HST data that were already available, and interpreted very loosely some upper limits from the published optical spectra. They thus determined a much higher oxygen abundance for TS 01 (however still holding the record of lowest $\mathrm{O} / \mathrm{H}$ found in $\mathrm{PNe}$ ). Using still deeper optical spectra together with the UV data, Stasińska et al. (2005) found, again, an oxygen abundance of about 1/100 that of the Sun, and provided estimates of the $\mathrm{C}$ and $\mathrm{N}$ abundances. 


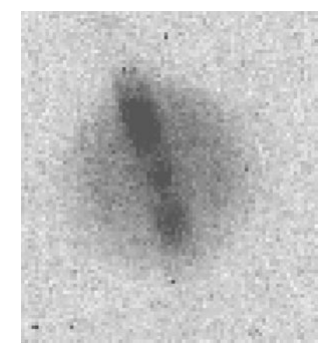

Figure 1. $\mathrm{H} \alpha$ image of TS 01 obtained with the Hubble Space Telescope.

Table 1. Chamical composition TS 01 obtained by various authors

\begin{tabular}{lcccccccc}
\hline $12+\log$ & T01 & R02 & J02 & PT05 & S05 & S09 & T09* & Sun \\
\hline $\mathrm{He} / \mathrm{H}$ & & & 10.82 & 10.91 & & $10.95 \pm 0.04$ & 10.95 & 10.93 \\
$\mathrm{C} / \mathrm{H}$ & & & $<8.30$ & $<8.0$ & $7.5 \pm 0.3$ & $8.0 \pm 0.3$ & $7.2 \pm 0.3$ & 8.4 \\
$\mathrm{~N} / \mathrm{H}$ & & & $<8.65$ & & $6.9 \pm .2$ & $7.2 \pm 0.3$ & $<6.9$ & 7.8 \\
$\mathrm{O} / \mathrm{H}$ & $6.3 \pm 0.5$ & $6.2 \pm 0.4$ & $6.93 \pm 0.4$ & $7.5 \pm 0.3$ & $6.85 \pm 0.25$ & $6.8 \pm 0.3$ & $<6.8$ & 8.7 \\
$\mathrm{Ne} / \mathrm{H}$ & & 5.9 & $7.47 \pm 0.4$ & $6.7 \pm 0.18$ & & $6.8 \pm 0.3$ & $6.5 \pm .3$ & 7.8 \\
$\mathrm{~S} / \mathrm{H}$ & & $<5.1$ & $<7.00$ & $<5.6$ & & $<5.7$ & & 7.1 \\
$\mathrm{Ar} / \mathrm{H}$ & & $<5.5$ & $<5.00$ & $<3.9$ & & $<4.7$ & & 6.2 \\
\hline
\end{tabular}

references: T01: Tovmassian et al. (2001); R02: Richer et al. (2002); J02: Jacoby et al. (2002); PT05: Péquignot \& Tsamis (2005); S05: Stasińska et al. (2005); S09: Stasińska et al. (2009); T09*: Tovmassian et al. (2009) (chemical composition derived from stellar atmosphere analysis).

\section{The most recent abundance determination in TS 01}

To definitely pin down the chemical composition of TS 01, Stasińska et al. (2009) obtained infrared observations of TS 01 with the Spitzer Space Telescope and produced several deep optical spectra of the nebula, one of them extracted from the Sloan Digital Sky Survey data base. In the meantime, Tovmassian et al. (2009) had obtained X-ray observations and conducted a spectroscopic and photometric analysis of the time variations of the central object, resulting in better description of the ionizing radiation. It turns out that the stellar spectrum is dominated by the cool component of the binary $\left(T_{\text {eff }} \simeq 60,000 \mathrm{~K}\right)$ below $40 \mathrm{eV}$, and by the hot component $\left(T_{\text {eff }} \simeq 180,000 \mathrm{~K}\right)$ above $40 \mathrm{eV}$ !

To minimize the errors due to geometry and aperture effects in the abundance determination, Stasińska et al. (2009) used the 3D photoionization code Cloudy_3D (Morisset 2006) to reproduce the $\mathrm{H} \alpha$ image of the nebula (Fig. 1) and properly take into account the slit sizes for each data set. The resulting abundances are listed under S09 in Table 1. Ironically, the error bars on the derived abundances are not smaller than in PT05 and S05 studies, despite the larger number of observational constraints and greater care in the modelling (suggesting that previous error bars were underestimated).

The nebular abundances of $\mathrm{C}, \mathrm{N}, \mathrm{O}$, and Ne in TS 01 are found to be, respectively, $1 / 3.5,1 / 4.2,1 / 70$, and $1 / 11$ of the Solar value, with typical uncertainties of a factor 2 . Thus the extreme $\mathrm{O}$ deficiency of this object is confirmed. The abundances of $\mathrm{S}$ and $\mathrm{Ar}$ are less than $1 / 30$ of Solar while He is very close to Solar.

From a stellar model analysis, some constraints could be obtained on the chemical composition of the progenitor star (see Table 1 under T09). They agree with the nebular results, which is extremely comforting, given the completely independent techniques. The only discrepancy concerns carbon, whose abundance is found lower in the star by 0.8 dex. So far, we have no clue on this issue. 

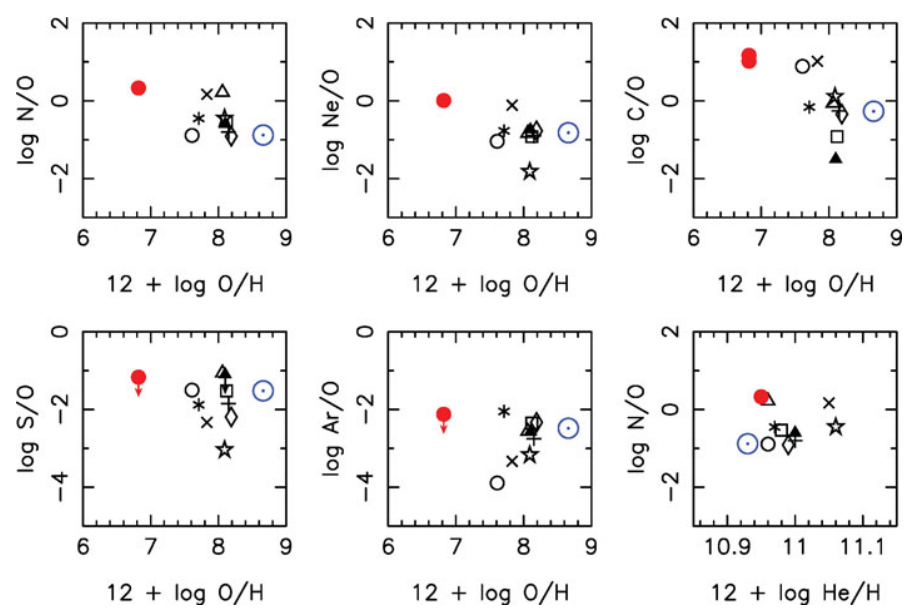

Figure 2. Comparison of TS 01 (filled red circle) with other Galactic PNe (black symbols) in abundance ratios diagrams. The Sun is represented by the Solar symbol.

\section{TS 01 as a probe for low-metallicity AGB nucleosyntesis}

In Fig. 2, the chemical composition of TS 01 is compared with that of other PNe in the halo. TS 01 is, by far, the most oxygen-poor. However, it is perhaps not the most metal-poor (see Fig. 17 of Stasińska et al. 2009). Yet, its metallicity is at most 1/30 of Solar, as indicated by the upper limits on the sulfur and argon abundances.

We have compared the abundance patterns of TS 01 with recent predictions for AGB nucleosynthesis using standard approaches (Karakas \& Lattanzio 2003, Cristallo et al. 2009). Standard low-mass AGB nucleosynthesis models show recurring 3rd dredge up episodes that increase the surface abundances of $\mathrm{C}, \mathrm{N}$ and $\mathrm{O}$. This is at odds with the $\mathrm{O}$ depletion seen in our object. Standard high-mass AGB models $\left(M_{\mathrm{ini}}>4 \mathrm{M}_{\odot}\right)$ do predict $\mathrm{O}$ depletion as a result of hot bottom burning during the TP-AGB phase, but they predict that $\mathrm{C}$ also should be depleted by the CNO-cycle in the envelope. More importantly, high-mass models are not relevant to TS 01 since the initial mass of the progenitor is estimated to be of $\simeq 1 \mathrm{M}_{\odot}$ (Tovmassian et al. 2009).

Rotation-induced mixing, introduced by Decressin et al. (2009) in AGB models of initial masses 2.5 and $3 \mathrm{M}_{\odot}$, efficiently transports chemical species from the H-burning shell to the surface during central He-burning and at second dredge-up. This decreases the $\mathrm{C}$ and $\mathrm{O}$ abundances and increases the $\mathrm{N}$ one (through the $\mathrm{CNO}$ cycle). $\mathrm{C}$ increases afterwards, during each 3rd dredge-up. In the case of TS 01, rotational transport may have been even strengthened by the stellar coupling in the binary system. We have therefore computed a model of a $1 \mathrm{M}_{\odot}$ star with metallicity corresponding to TS 01 and initial rotation of $100 \mathrm{~km} \mathrm{~s}^{-1}$.

Fig. 3 shows the result of this model and of a similar model without rotation, and compares it to the chemical composition of TS 01. It can be seen that rotation greatly improves the comparison. However the $\mathrm{O}$ depletion in the model is only consistent with the upper limit allowed by the observations. The agreement can be improved for a higher initial velocity, for example as the result of mass-transfer when the massive primary star expands. This high velocity can also lead to a larger enhancement of $\mathrm{Ne}$ as required by the observations. In this way the full observational pattern of TS 01 can be explained, except for the He abundance which is somewhat higher than observed. 

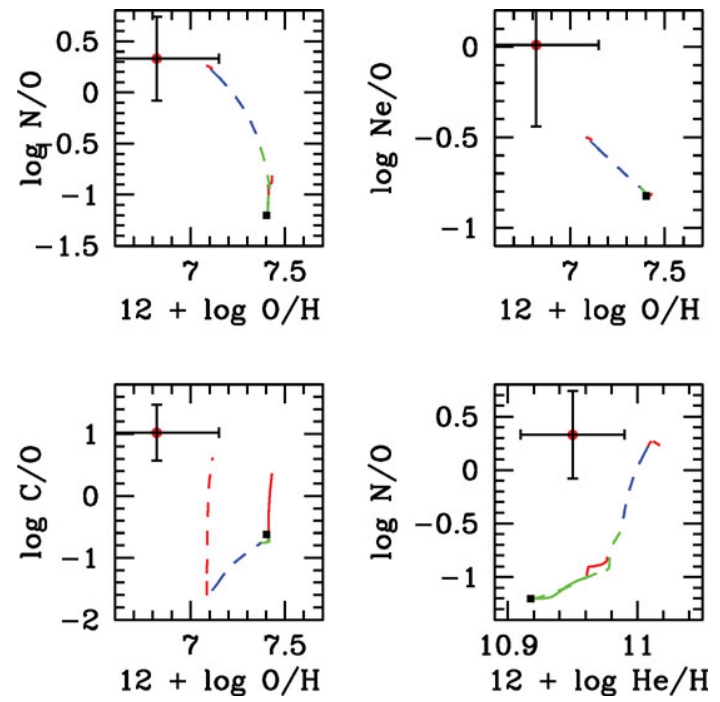

Figure 3. Comparison of the chemical composition in TS 01 with the results of stellar evolution models for a star with initial mass $1 \mathrm{M}_{\odot}$, and initial composition as indicated by the black dot. Continuous line: without rotation; Dashed line: with rotation.

\section{Conclusion}

The planetary nebula TS 01 has been confirmed as the most oxygen-poor among known $\mathrm{PNe}$, with an oxygen abundance of $1 / 70$ of the Solar value. From upper limits on its S and Ar abundances, its metallicity is estimated to be at most 1/30 of Solar.

Standard AGB models with initial masses and metallicities corresponding to the progenitor of TS 01 are not able to reproduce the observed abundance pattern. Taking into account stellar rotation with an initial velocity of $100 \mathrm{~km} \mathrm{~s}^{-1}$, as we have done in a model built for this purpose, greatly improves the situation. In TS 01, one may expect even faster rotation, due to mass transfer in the binary, therefore bringing model results and observations in even better agreement.

\section{Acknowledgements}

Many thanks to M. Richer, M. Peña, R. Szczerba, C. Charbonnel, L. Yungelson, R. Napiwotzki, S. Simon-Diaz, L. Jamet, Y. Izotov, A. Fullerton, V. Suleimanov, J. Tomsick who provided important input at various stages in our study of TS 01.

\section{References}

Cristallo, S., Straniero, O., Gallino, R. et al., 2009, ApJ, 696, 797

Decressin, T., Charbonnel, C., Siess, L. et al., 2009, arXiv:0907.5200

Jacoby, G. H., Feldmeier, J. J., Claver, C. F., et al. 2002, AJ, 124, 3340

Karakas, A. I. \& Lattanzio, J. C. 2003, PASA, 20, 393

Morisset C., 2006, IAUS, 234, 467

Péquignot, D. \& Tsamis, Y. 2005, A\&A, 430, 187

Richer, M. G., Tovmassian, G., Stasińska, G. et al., 2002, A\&A, 395, 929

Stasińska, G., Tovmassian, G. H., Richer, et al., 2005, IAUS, 228, 323

Stasińska et al. 2009, $A \mathscr{E} A$ in press

Tovmassian, G. H., Stasińska, G., Chavushyan, V. H., et al., 2001, A\&A, 370, 456

Tovmassian, G. H., Napiwotzki, R., Richer, M. G., et al., 2004, ApJ, 616, 485

Tovmassian et al. 2009, ApJ submitted 